\title{
IMPACTOS GERADOS PELO USO DE CLOROQUINA, HIDROXICLOROQUINA E AZITROMICINA COMO TRATAMENTO DE PACIENTES COM COVID-19
}

\author{
IMPACTS GENERATED BY THE USE OF CHLOROQUINE, \\ HYDROXYCHLOROQUINE AND AZITHROMYCIN AS TREATMENT OF \\ PATIENTS WITH COVID-I9
}

\author{
Fabio José da Silva Melo
}

Carlos Laubert Nunes Lemos ${ }^{2}$

Alexandre Torres Vasconcelos ${ }^{3}$

Marcos Paulo Santos Passos ${ }^{4}$

RESUMO: Em razão da gravidade do cenário mundial assolado pela pandemia do coronavírus, foram iniciados testes de medicamentos com o intuito de encontrar a solução mais eficaz e segura para o tratamento da COVID-19. Em estudos preliminares, fármacos como a cloroquina e hidroxicloroquina associados à azitromicina tiveram atividade antiviral contra o SARS-CoV-2. Perante o exposto, o presente trabalho teve como objetivo avaliar os impactos do uso da cloroquina, da hidroxicloroquina e da azitromicina, no tratamento de pacientes com COVID-ı. Para isso, foi realizada uma revisão sistemática de literatura de caráter descritivo em diversas bases de dados, com o intervalo temporal entre 2019 e 202I, utilizando os descritores em ciências da saúde (DeCS): Covid-I9, Sars-CoV-2, Hydroxychloroquine, Chloroquine e Azithromycin, utilizando o operador booleano "AND”. Após a análise dos artigos, constatou-se que a cloroquina, hidroxicloroquina e azitromicina não possuem eficácia no tratamento ao COVID-ı e comprometem o ritmo cardíaco dos pacientes em decorrência dos efeitos colaterais existentes durante o tratamento.

Palavras- Chaves: Covid-ı9, Sars-CoV-2, Hydroxychloroquina, Chloroquina, Azithromycina.

\footnotetext{
${ }^{1}$ Universidade Salvador - UNIFACS, Feira de Santana - Bahia. E-mail: Fabiinhomelloo@hotmail.com

${ }^{2}$ Universidade Salvador - UNIFACS, Feira de Santana - Bahia. , carlos_laubert@hotmail.com

${ }^{3}$ Universidade Salvador - UNIFACS, Feira de Santana - Bahia.E_mail: atvouxtv@gmail.com, ORCID: https://orcid.org/oooo-0oo2-6028-3722

${ }^{4}$ Especialista em análises clínicas e citopatologia clínica. Universidade Salvador - UNIFACS. ORCID: https://orcid.org/oooo-ooor-9076-4650
} 
ABSTRACT: Due to the seriousness of the world scenario devastated by the coronavirus pandemic, drug testing was started in order to find a more effective and safe solution for the treatment of COVID-I9. In preliminary studies, drugs such as chloroquine and hydroxychloroquine associated with azithromycin had antiviral activity against SARS-CoV-2. Given the above, this study aimed to evaluate the impacts of the use of chloroquine, hydroxychloroquine and azithromycin in the treatment of patients with COVID-19. For this, a systematic literature review of a descriptive nature was carried out in several databases, with the time interval between 2019 and 2021, using the descriptors in health sciences (DeCS): Covid-19, Sars-CoV-2, Hydroxychloroquine, Chloroquine and Azithromycin, using the Boolean operator "AND". After analyzing the articles, it was found that chloroquine, hydroxychloroquine and azithromycin did not bear efficiency in the treatment of COVID-ig and that the patients' heart rhythm was compromised as a result of the effects existing during the treatment.

Keywords: Covid-19, Sars-CoV-2, Hydroxychloroquine, Chloroquine, Azithromycin.

\section{INTRODUÇÃO}

O SARS-CoV-2 é um vírus que faz parte da família Coronaviridae, os vírus dessa família afetam aves e mamíferos, causando doenças, no caso dos seres humanos ele é responsável por causar infecções do trato respiratório podendo levar a quadros leves ou graves dependendo da variante do vírus (VIEIRA; EMERY; ANDRIOLO, 2020).

No final de dezembro de 2019, a Organização Mundial de Saúde (OMS) foi notificada com vários casos de pneumonia na cidade de Wuhan, província de Hubei, na China. Em 7 de janeiro de 2020 as autoridades chinesas confirmaram que haviam identificado um novo tipo de coronavírus, o sequenciamento genético sugeriu ser um betacoronavírus ligado ao vírus da síndrome respiratória aguda grave (SARS), nomeado temporariamente como $2019-\mathrm{nCoV}$ e posteriormente intitulado de SARS-CoV-2. Em II de março de 2020 a síndrome respiratória severa do coronavírus 2 (Sars-Cov-2) foi caracterizada pela Organização Mundial de Saúde como uma pandemia (SOUZA et al., 2021).

Após a entrada do vírus no organismo humano ele adere a mucosa e liga-se à enzima $\mathrm{ECA}_{2}$ proteína que medeia a entrada do vírus na célula-alvo, depois do 
reconhecimento, o envelope viral funde-se à membrana citoplasmática do hospedeiro, permitindo a entrada dele no citosol da célula, uma vez no endossomo, segue para o citoplasma e libera o ácido ribonucleico (RNA) viral de fita simples com sentido positivo, permitindo a produção de poliproteínas e estruturas proteicas, o que dá início ao processo de replicação viral (BRITO et al., 2020).

Mesmo não havendo informações suficientes sobre o vírus, medidas emergenciais foram adotadas, buscando conter a disseminação, como o uso obrigatório de máscaras e o distanciamento social. A transmissão do SARS-CoV-2 pode ocorrer através do contato direto, indireto ou próximo com pessoas infectadas através de secreções infectadas como saliva e secreções respiratórias ou de suas gotículas respiratórias, que são expelidas quando uma pessoa infectada tosse, espirra, fala ou canta (OPAS, 2020).

Devido à gravidade das complicações e o poder fatal da COVID-ı9, foram iniciados diversos ensaios e testes com medicamentos para encontrar terapêuticas mais eficazes e acessíveis. Dados preliminares de estudos in vitro identificaram atividade antiviral dos fármacos cloroquina e hidroxicloroquina, associados a antibióticos macrolídeos como azitromicina, e estes foram recomendados em alguns países como terapia medicamentosa contra o SARS-CoV-2. Apesar desses fármacos serem indicados para outras doenças, o uso nesta pandemia é experimental, e mesmo o uso compassivo pode representar riscos à saúde devido ao potencial de causar reações adversas, principalmente o risco de cardiotoxicidade (MELO et al., 202I).

Considerando a necessidade de uma alternativa terapêutica para o tratamento de pacientes com COVID-I9, o presente estudo teve como objetivo realizar uma revisão sistemática de literatura sobre os impactos gerados pelo uso da cloroquina, hidroxicloroquina e azitromicina, como tratamento de pacientes com COVID-i9, abordando as evidências científicas existentes até o presente momento. 


\section{MATERIAIS E MÉTODOS}

O presente trabalho trata-se de uma revisão de literatura do tipo sistemática de caráter descritivo, com abordagem qualitativa que pretende retratar as consequências geradas com o uso da cloroquina, hidroxicloroquina e azitromicina no tratamento de pacientes com COVID-ı9, sendo assim, foram realizadas pesquisas por informações relevantes acerca do assunto, reunindo dados fundamentados sobre o tema.

Foram utilizadas as seguintes bases de dados: SciElo (Scientific Electronic Library Online), LILACS (Literatura Latino-Americana e do Caribe em Ciências da Saúde) e PubMed (National Center for Biotechnology Information - NCBI), utilizando AND como operador booleano e tendo os seguintes descritores em ciências da saúde (DeCS): Covid-ı9, Sars-CoV-2, Hydroxychloroquine, Chloroquine e Azithromycin.

Foram filtradas publicações entre 2019 e 2021, com idiomas em português, inglês e espanhol. Foram excluídos artigos em duplicata e artigos que não apresentaram concordância com o tema.

\section{RESULTADOS}

Com base no que foi observado por meio da análise prévia dos trabalhos publicados no período de 2019 a 202I, foram identificados um total de II2 publicações, visto que, foram encontrados 12 no SciElo, 85 no Lilacs e 15 no PubMed.

Após a investigação da temática e aplicação da filtragem contendo "ano" e "idioma", foi realizada uma triagem com o intuito de retirar artigos em duplicata, excluir artigos pela leitura do título e/ou resumo e excluir artigos após a leitura do texto completo por divergências com o tema.

Após a triagem foram selecionados 7 artigos, sendo i no SciElo, i no Lilacs e 5 no PubMed, os quais foram escolhidos por apresentarem maior relevância e similaridade com o conteúdo abordado, observando que a maioria dos estudos se dedicou a demonstrar a eficácia e a segurança da cloroquina, hidroxicloroquina e azitromicina 
utilizados no tratamento da COVID-I9, realizando levantamentos dos possíveis desencadeamentos de novas enfermidades associadas aos medicamentos que elevam os riscos de saúde dos pacientes. O detalhamento da metodologia empregada na seleção e inclusão dos estudos está presente no fluxograma da figura I.

$\mathrm{Na}$ Tabela I estão elencados os títulos dos artigos selecionados, seus autores, o ano da publicação e o tipo de estudo realizado.

Figura I. Fluxograma da metodologia empregada na seleção dos artigos presentes nesta revisão.

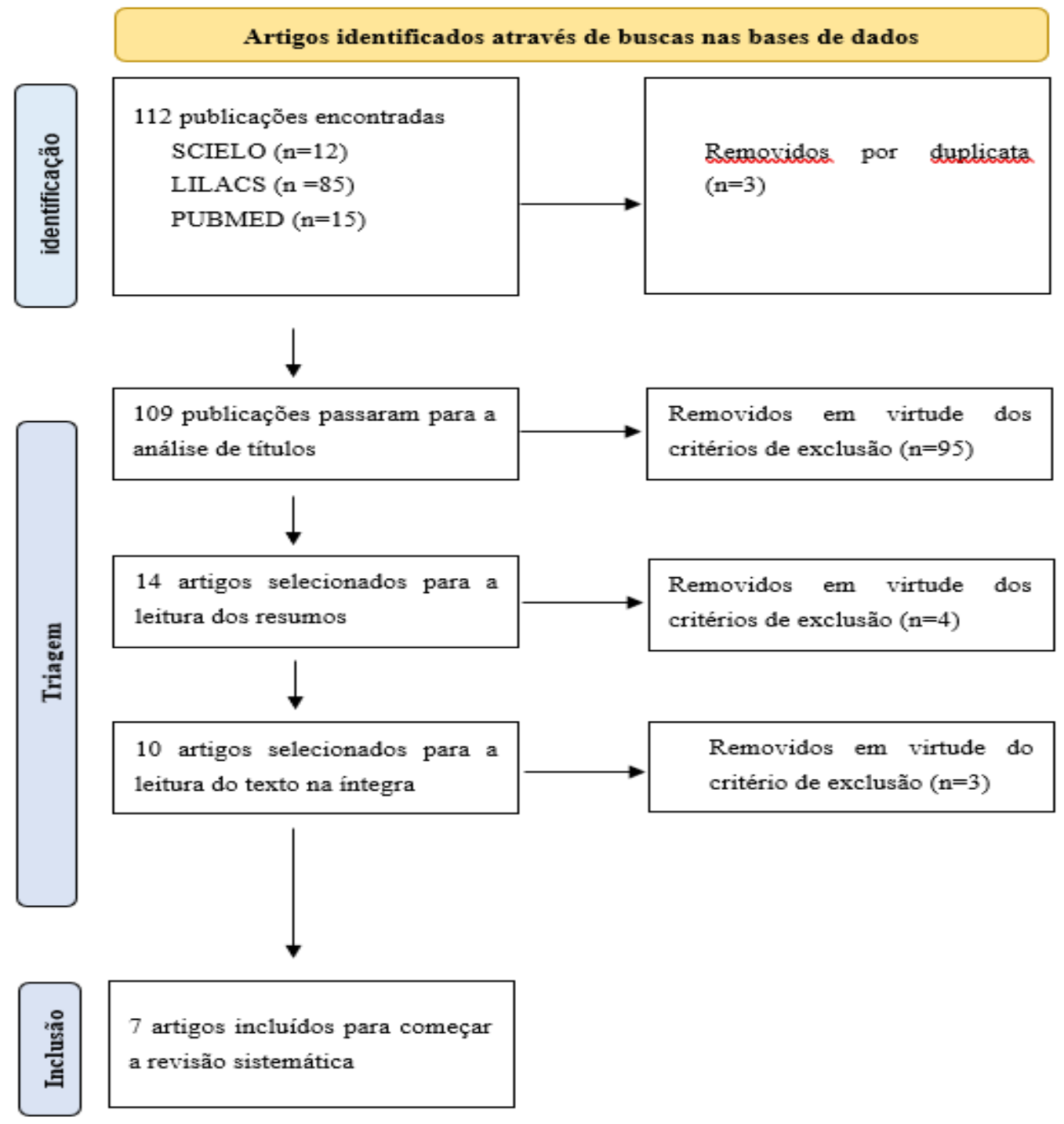


TABELA r. Apresentação dos artigos analisados na revisão segundo tema, autor, ano e tipo de estudo.

\begin{tabular}{|c|c|c|c|}
\hline Ordem & Título & Autor (es) & Tipo de Estudo \\
\hline $\mathrm{I}^{\mathbf{O}}$ & $\begin{array}{l}\text { Reações adversas a medicamentos em pacientes com COVID-I9 no Brasil: } \\
\text { análise das notificações espontâneas do sistema de farmacovigilância } \\
\text { brasileiro }\end{array}$ & $\begin{array}{l}\text { MELO et al., } \\
\text { 202I }\end{array}$ & $\begin{array}{l}\text { Estudo transversal, com etapas descritiva- } \\
\text { exploratória e analítica }\end{array}$ \\
\hline $2^{\circ}$ & $\begin{array}{l}\text { Effect of High vs Low Doses of Chloroquine Diphosphate as Adjunctive } \\
\text { Therapy for Patients Hospitalized With Severe Acute Respiratory } \\
\text { Syndrome Coronavirus } 2 \text { (SARS-CoV-2) Infection: A Randomized } \\
\text { Clinical Trial }\end{array}$ & $\begin{array}{l}\text { BORBA et al., } \\
2020\end{array}$ & $\begin{array}{l}\text { Ensaio clínico randomizado, duplo-cego, de três } \\
\text { braços }\end{array}$ \\
\hline $3^{\circ}$ & $\begin{array}{l}\text { Assessment of COVID-ig Treatment containing both } \\
\text { Hydroxychloroquine and Azithromycin: A natural clinical trial }\end{array}$ & $\begin{array}{l}\text { ABBAS et al., } \\
2020\end{array}$ & Ensaio clínico natural sem grupo controle \\
\hline $4^{\circ}$ & $\begin{array}{l}\text { Hydroxychloroquine with or without Azithromycin in Mild-to-Moderate } \\
\text { Covid-I9 }\end{array}$ & $\begin{array}{l}\text { CAVALCAN } \\
\text { TI et al., } 2020\end{array}$ & $\begin{array}{l}\text { Ensaio clínico multicêntrico, randomizado, aberto, } \\
\text { de três grupos e controlado }\end{array}$ \\
\hline $5^{0}$ & $\begin{array}{l}\text { Hydroxychloroquine with azithromycin in patientshospitalized for } \\
\text { mild and moderate COVID-i9 }\end{array}$ & $\begin{array}{l}\text { LAMBACK et } \\
\text { al., 2021 }\end{array}$ & $\begin{array}{l}\text { Estudo comparativo retrospectivo com análise de } \\
\text { prontuários médicos. }\end{array}$ \\
\hline 60 & $\begin{array}{l}\text { Association of Treatment With Hydroxychloroquine or Azithromycin } \\
\text { With In-Hospital Mortality in Patients With COVID-19 in New York } \\
\text { State }\end{array}$ & $\begin{array}{l}\text { ROSENBEG } \\
\text { et al., } 2020\end{array}$ & Estudo de coorte multicêntrico retrospectivo \\
\hline $7^{0}$ & $\begin{array}{l}\text { Hydroxychloroquine with or without azithromycin for treatment } \\
\text { of early SARS-CoV-2 infection among high-risk outpatient adults: } \\
\text { A randomized clinical trial }\end{array}$ & $\begin{array}{l}\text { JOHNSTON et } \\
\text { al., 2021 }\end{array}$ & $\begin{array}{l}\text { Ensaio clínico randomizado, duplo-cego, de três } \\
\text { braços }\end{array}$ \\
\hline
\end{tabular}




\section{DISCUSSÃO}

Diante dos resultados encontrados os artigos selecionados abordaram o conteúdo de forma desejada, contribuindo com o interesse da revisão. Nesse contexto foram encontrados estudos que avaliassem casos de pacientes por meio de software e ensaios clínicos com o objetivo de avaliar a eficácia e segurança dessa terapia farmacológica. Antes de apresentar os efeitos desses medicamentos, é necessário compreender mais sobre o mecanismo de ação destes contra o SARS-CoV-2.

Estudos envolvendo a cloroquina sugerem que ela exerce um efeito antiviral durante as condições pré e pós-infecção, interferindo na glicosilação da ECA2 e bloqueando a fusão do vírus com a célula hospedeira. A glicosilação terminal do $\mathrm{ECA}_{2}$ prejudicada pode reduzir a eficiência de ligação entre o ECA2 nas células hospedeiras e a proteína spike do SARS-CoV-2 (AGONDI; AUN; GIAVINABIANCHI, 2020).

A hidroxicloroquina pode aumentar o $\mathrm{pH}$ intracelular e inibir a atividade lisossômica das células apresentadoras de antígenos, incluindo células dendríticas e células $\mathrm{B}$, impedindo o processamento de antígenos. Esse processo limita a ativação das células $\mathrm{T}$, a diferenciação e expressão de proteínas coestimuladoras e as citocinas produzidas por células T e células B (AGONDI; AUN; GIAVINA-BIANCHI, 2020).

Estudos apontam que a hidroxicloroquina também age no citoplasma, interferindo na interação de DNA e síntese de ácidos nucleicos virais, atenuando a resposta pró-inflamatória e geração de citocinas. Os dois mecanismos indicam que a hidroxicloroquina tem a habilidade de suprimir a SARS-CoV-2 por inibir a hiperativação do sistema imune desencadeada pelo vírus e, consequentemente, diminuir a progressão da doença de leve a severa, porém ainda sem comprovação clínica (MENEZES; SANCHES; CHEQUER, 2020).

Azitromicina, antimicrobiano derivado da classe dos macrolídeos, demonstrou ser ativo in vitro contra os vírus Zika e Ebola, além disso, esse fármaco apresentou eficácia na prevenção da evolução para quadros mais graves de infecções 
virais do trato respiratório. Alguns estudos apontaram que, além de sua atividade antimicrobiana de impedir uma superinfecção bacteriana, atenuando assim uma infecção viral causada pelo SARS COV-2, os macrolídeos apresentam propriedades anti-inflamatórias e antivirais (VANZELER et al., 2021).

No primeiro artigo, de Melo et al. (2021), onde foi realizada a pesquisa utilizando como fonte de dados os relatórios de segurança de casos individuais (ICSR, em inglês) do programa cedido pela Agência Nacional de Vigilância Sanitária (Anvisa), teve por objetivo avaliar as reações adversas a medicamentos (RAMs) nos pacientes com COVID-I9 identificadas entre I de março e I5 agosto de 2020 no Brasil. Foram identificadas 63I RAMs em 402 pacientes, sendo que a maioria dos pacientes foi do sexo masculino. A faixa etária mais atingida foi a de 45-64 anos. Os medicamentos que mais apresentaram RAMs foram a hidroxicloroquina (59,5\%), azitromicina $(9,8 \%)$ e a cloroquina $(5,2 \%)$, sendo que a hidroxicloroquina e a cloroquina foram os dois únicos medicamentos que estiveram estatisticamente associados à presença de RAM grave. Pacientes que usaram a hidroxicloroquina registraram quase duas vezes mais chances de apresentarem RAMs graves do que aqueles que não usaram este medicamento, de uma forma mais agravada, os que fizeram o uso da cloroquina registraram quase seis vezes mais chances de apresentarem RAMs graves comparados aos que não usaram este medicamento. Dentre as RAMs a mais relatada foi o prolongamento do intervalo QT, com um percentual de 212 registros (33,6\%), sendo que in casos foram classificados com fatais/óbitos.

Acredita-se que os medicamentos em questão exercem uma potencialização em uma determinada ação no sistema cardivascular, essa associação pode estar atrelada a uma função similiar desempenhada pelos fármacos, "ambos bloqueiam o canal de potássio hERG, podendo prolongar a repolarização ventricular e causar torsades de pointes" (Wu et al., 202I).

De acordo com estudos realizados por Borba et al. (2021), foram divulgados os resultados preliminares de um ensaio clínico denominado CloroCovid-ig que teve como objetivo avaliar a segurança e secundariamente a eficácia de duas doses 
diferentes de cloroquina como terapia complementar para pacientes hospitalizados com síndrome do desconforto respiratório causada pelo COVID-19. Foram incluídos 8I casos no ensaio, divididos em 4I no grupo de alta dose e 40 no grupo de baixa dose, foi necessário que a equipe suspendesse o ensaio no sexto dia devido à ocorrência de vários casos em ambos os grupos envolvendo um número significativo de reações adversas graves e mortes. Houve ir mortes ao total no estudo, 7 no grupo de alta dose e 4 no grupo de baixa dose. O percentual de mortalidade neste estudo foi de $13,5 \%$ e no momento da suspensão não houve diferenças estatisticamente significativas entre os grupos. Um prolongamento do intervalo QT de mais de $500 \mathrm{~ms}$ foi observado em $17,9 \%$ dos pacientes em ambos os grupos de estudo, sugerindo que o uso de cloroquina no tratamento da COVID-ı9 não deve ser recomendada devido a questões de segurança em relação ao prolongamento do intervalo QT e aumento da letalidade.

Um estudo realizado por ABBAS et al. (2020) teve por objetivo avaliar a eficácia clínica e o perfil de segurança do protocolo de tratamento do COVID-ı9 contendo hidroxicloroquina e azitromicina. O estudo em questão trata-se de um ensaio clínico natural sem grupo controle, realizado em um hospital especializado no Iraque. $\mathrm{O}$ estudo incluiu I6I pacientes que foram admitidos com RT-PCR positivo e sintomas clínicos de COVID-19. A duração do tratamento foi de 14 dias. O regime padrão de tratamento da COVID-ı que incluía hidroxicloroquina e azitromicina teve um efeito positivo significativo na temperatura corporal dos pacientes, tosse, falta de ar, frequência respiratória, frequência de pulso e nos níveis de glicose em jejum. As reações adversas aos medicamentos que os pacientes apresentaram durante o tratamento foram dores de estômago/ cólicas, hipoglicemia, dores de cabeça, alterações de humor, tonturas, coceira, diarreia, fraqueza nos músculos, náusea, erupção cutânea, vômito, prolongamento do intervalo QT, arritmia e conjuntivite, entretanto somente arritmia, prolongamento do intervalo QT e conjuntivite foram provavelmente causadas pelos dois medicamentos, as outras reações adversas relatadas durante o tratamento provavelmente devem ter sido causadas pela COVID-ı. O ensaio clínico comprovou a eficácia da hidroxicloroquina e azitromicina no tratamento ao COVID-I9, o tratamento reduziu os sinais e sintomas 
manifestados pelos pacientes, entretanto também houve a presença de alguns efeitos colaterais decorrentes dos medicamentos durante o tratamento, apresentando riscos principalmente ao ritmo cardíaco dos pacientes.

Mesmo que a diferença entre o uso isolado de cada medicamento não seja muito significativa, o uso concomitante de ambos está associado a uma mudança maior no prolongamento do intervalo QT, elevando a possibilidade de consequências mais danosas à saúde do paciente, como origem de arritmias e morte súbita, mesmo em poucas ocasiões (JUSTINA et al., 2020).

É interessante observar que alguns fatores como a variação nos padrões de dosagem e na duração do tratamento para cada medicamento pode influenciar potencialmente no momento em que o eletrocardiograma com o QT mais longo pode ser observado (RAMIREDDY et al., 2020).

Um ensaio clínico multicêntrico com 504 pacientes admitidos, executado em 55 hospitais do Brasil, realizado por CAVALCANTI et al. (2020) com o objetivo de avaliar a segurança e eficácia da hidroxicloroquina e azitromicina no tratamento ao Covid-ı9 em comparação ao tratamento padrão, determinou que não houveram diferenças significativas no resultado do estado clínico entre os grupos que receberam hidroxicloroquina sozinha, hidroxicloroquina com azitromicina e o tratamento padrão, porém a prolongação do intervalo de QT foi manifestada em pacientes que receberam hidroxicloroquina associada à azitromicina ou hidroxicloroquina sozinha mais do que naqueles que receberam azitromicina sozinha ou nenhum dos medicamentos em estudo. Os pacientes foram designados aleatoriamente em uma proporção de I: I: I para receber tratamento padrão, tratamento padrão mais hidroxicloroquina na dose de $400 \mathrm{mg}$ duas vezes ao dia, ou tratamento padrão mais hidroxicloroquina na dose de $400 \mathrm{mg}$ duas vezes ao dia mais azitromicina na dose de $500 \mathrm{mg}$ uma vez ao dia durante 7 dias. Portanto, ao final do estudo, que teve duração de is dias, ficou evidente que o uso de hidroxicloroquina sozinha ou com azitromicina não melhorou o estado clínico dos pacientes, entretanto os efeitos colaterais dos medicamentos causaram riscos ao ritmo cardíaco dos pacientes. 
O risco potencial especulado de prolongamento QT grave induzido pela associação dos dois medicamentos ainda não foi estabelecido, mas deve ser considerado. Quanto a cada tratamento, o custo-benefício do risco deve ser avaliado individualmente (GAUTRET et al., 2020).

Com base em um estudo comparativo retrospectivo com análise de dados eletrônicos de prontuários médicos de pacientes admitidos por COVID-i9 leve e moderada realizado por LAMBACK et al. (202I) que teve por objetivo avaliar a eficácia da hidroxicloroquina em combinação com azitromicina em termos de resultados clínicos e bioquímicos em pacientes adultos com COVID-I9 hospitalizados em decorrência da síndrome do desconforto respiratório agudo (SDRA), a terapêutica adotada com uso de hidroxicloroquina e azitromicina no combate ao COVID-19 não obteve efeitos expressivos em relação ao quadro clinico dos pacientes, tendo resultados semelhantes ao tratamento exercido no combate a COVID-I9 do grupo controle (tratamento padrão). Portanto o tratamento com hidroxicloroquina e azitromicina demostrou não ser eficaz. O estudo foi realizado em um hospital privado quaternário do Rio de Janeiro no Brasil, envolvendo 193 pacientes adultos hospitalizados por COVID-ı9 leve e moderada. Dos 193 pacientes, IOI $(52,3 \%)$ usavam hidroxicloroquina associada a azitromicina (grupo ativo) e 92 $(47,7 \%)$ não, sendo considerado como o grupo controle. O tratamento consistiu em $400 \mathrm{mg}$ duas vezes ao dia de hidroxicloroquina no primeiro dia e $400 \mathrm{mg}$ uma vez ao dia durante os próximos 4 dias, associada à azitromicina $500 \mathrm{mg}$ por dia também por cinco dias.

Em outra abordagem, a pesquisa de ROSENBERG et al. (2020) que consistiu em um estudo de coorte multicêntrico retrospectivo de pacientes de uma amostra aleatória de todos os pacientes internados com COVID-i9 confirmado por laboratório em 25 hospitais de New York, nos Estados Unidos, teve o objetivo de descrever a associação entre o uso de hidroxicloroquina com ou sem azitromicina e resultados clínicos entre pacientes hospitalizados com diagnóstico de COVID-I9. Dados dos prontuários dos pacientes foram analisados, dos 1438 pacientes participantes do estudo, 735 (51,1\%) receberam hidroxicloroquina associada à 
azitromicina, 27I (18,8\%) receberam apenas hidroxicloroquina, 2II (I4,7\%) receberam apenas azitromicina e 221 $(15,4 \%)$ não receberam nenhuma droga. Uma proporção maior de pacientes recebendo hidroxicloroquina associada à azitromicina sofreu parada cardíaca $(15,5 \%)$ e achados de eletrocardiograma anormais como arritmias e prolongamento do intervalo de $\mathrm{QT}$ (27,1\%), assim como aqueles pacientes que receberam apenas hidroxicloroquina (13,7\% e 27,3, respectivamente), comparado com pacientes que receberam azitromicina sozinha (6,2\% e $16,1 \%$, respectivamente) e nenhuma droga $(6,8 \%$ e $14,0 \%$, respectivamente). Pacientes que receberam hidroxicloroquina associada à azitromicina $(30,7 \%)$ e hidroxicloroquina sozinha (19,2\%) tiveram níveis mais elevados de admissão à UTI do que aqueles que receberam azitromicina sozinha (I0,9\%) e nenhuma droga (I2,2\%). A mortalidade hospitalar foi de 22,5\% com o uso de hidroxicloroquina associada à azitromicina, I8,9\% com o uso de hidroxicloroquina sozinha, I0,9\% com o uso de azitromicina sozinha e $17,8 \%$ com o uso de nenhuma droga. Ao final do estudo foi constatado através da mortalidade hospitalar que a hidroxicloroquina e azitromicina não possuem eficácia contra a COVID-I9, também ficou explícito que o uso de hidroxicloroquina e azitromicina, associadas ou não, influencia diretamente no agravo da frequência cardíaca e do estado clínico dos pacientes.

Um ensaio clínico randomizado, duplo-cego, de três braços (I: I: I) realizado por JOHNSTON et al. (202I) em cinco hospitais dos Estados Unidos, que envolveu I52 pacientes ambulatoriais adultos com infecção por SARS-CoV-2, teve por objetivo avaliar se as opções de tratamento para pacientes ambulatoriais com COVID-I9 podiam reduzir a morbidade e prevenir a transmissão da SARS-CoV-2. Os participantes foram aleatoriamente designados para receber o tratamento, foi administrado para 49 participantes a hidroxicloroquina $400 \mathrm{mg}$ duas vezes ao dia no primeiro dia, seguido por $200 \mathrm{mg}$ duas vezes ao dia durante 9 dias. Para $5 \mathrm{I}$ participantes, além da hidroxicloroquina, também foi administrado a azitromicina $500 \mathrm{mg}$ no primeiro dia, em seguida $250 \mathrm{mg}$ durante 9 dias e o restante dos 52 participantes fizeram parte do grupo controle. Ao final do estudo ficou comprovado que a hidroxicloroquina sozinha ou administrada junto com a azitromicina não 
agilizou a resolução dos sintomas da COVID-ı́ e não acelerou a depuração viral em comparação com o grupo controle demonstrando, portanto, que a hidroxicloroquina e azitromicina não possuem eficácia contra o SARS-CoV-2.

Após a realização desta revisão de literatura, pode-se destacar que o tratamento de pacientes com COVID-ig utilizando cloroquina, hidroxicloroquina e azitromicina, não possui eficácia e segurança. A utilização desses fármacos no tratamento da COVID-ı9 é excessivamente prejudicial ao ritmo cardíaco, causando o prolongamento do intervalo de QT e, consequentemente, podendo induzir o óbito.

\section{REFERÊNCIAS}

ABBAS, H. M.; Al-JUMAILI, A. A.; NASSIR, K. F. et al. (2020). Assessment of COVID-19 Treatment containing both Hydroxychloroquine and Azithromycin: A natural clinical trial. International Journal of Clinical Practice. v. 75, n. 9 p. I-9. https://doi.org/ro.IIII/ijcp.13856

AGONDI, R. C.; AUN, M. V.; GIAVINA-BIANCHI, P. (2020). COVID-i9, enzima conversora da angiotensina 2 e hidroxicloroquina. Arquivos de Asma, Alergia e Imunologia. v. 4, n. I, p. 138-I40. https://doi.org/10.5935/2526-5393.20200018

BORBA, M. G. S.; VAL, F. F. A.; SAMPAIO, V. S. et al. (2020). Chloroquine diphosphate in two different dosages as adjunctive therapy of hospitalized patients with severe respiratory syndrome in the context of coronavirus (SARS-CoV-2) infection: Preliminary safety results of a randomized, double-blinded, phase IIb clinical trial (CloroCovid-I9 Study). MedRxiv. v. 2, p. I-3o. https://doi.org/Io.IooI/jamanetworkopen.2020.8857

BRITO, S. B. P.; Braga, O. I.; MORAES, M. M.; et al. (2020). Immunopathological mechanisms involved in SARS-CoV-2 infection. Jornal Brasileiro de Patologia e Medicina Laboratorial. v. 56.https://doi.org/10.5935/1676-2444.20200056

CAVAlCANTI, A. B.; ZAMPIERI, F. G.; ROSA, R. G. et al. (2020). Hydroxychloroquine with or without Azithromycin in Mild-to-Moderate Covid-rg. The new england journal of medicine. v. 383, n. II, p. 2.04I-2.052. https://doi.org/Io.1056/NEJMoa2019014 
GAUTRET, P.; LAGIER, J.; PAROLA, P. et al. (2020). Hydroxychloroquine and azithromycin as a treatment of COVID-r9: results of an open-label non-randomized clinical trial. International Journal of Antimicrobial. v. 56, n. $7 . \quad$ p. 2-7. https://doi.org/ro.ıor6/j.ijantimicag.2020.105949

JOHNSTON, C.; BROWN, E. R.; STEWART, J. et al. (2021). Hydroxychloroquine with or without azithromycin for treatment of early SARSCoV-2 infection among high-risk outpatient adults: A randomized clinical trial. EClinicalMedicine. v. 33. https://doi.org/ro.1016/j.eclinm.2021.100773

JUSTINA, B. G. D.; AQUINO, C. M.; LADEIA. D. N. et al. (2020). Prevalência de arritmias em pacientes com Sars-cov-2 tratados com hidroxicloroquina e azitromicina. Brazilian Journal of Health Review. v. 3, n. 4, p. 10876-10890. https://doi.org/ro.34119/bjhrv3n4-344

LAMBACK, E. B.; OLIVEIRA. M. A.; HADDAD. A. F. et al. (2021). Hydroxychloroquine with azithromycin in patients hospitalized for mild and moderate COVID-19. Brazilian Journal of Infectious Diseases. v. 25, n. 2. https://doi.org/ro.ıor6/j.bjid.2021.101549

MELO, J. R. R.; DUARTE, E. C.; MORAES, M. V. et al. (2021). Reações adversas a medicamentos em pacientes com COVID-ı9 no Brasil: análise das notificações espontâneas do sistema de farmacovigilância brasileiro. Cadernos de Saúde Pública. v. 37, n. I. https://doi.org/10.1590/oro2-311X00245820

MENEZES, C. R.; SANCHES, C.; CHEQUER, F. M. D. (2020). Efetividade e toxicidade da cloroquina e da hidroxicloroquina associada (ou não) à azitromicina para tratamento da COVID-19. O que sabemos até o momento?. Journal of Health dr Biological Sciences. v. 8, n. I, p. I-9. http://dx.doi.org/I0.12662/23173076jhbs.v8ir.3206.pi-9.2020

Organização Pan-Americana da Saúde (2020). Transmissão do SARS-CoV-2: implicações para as precauções de prevenção de infecção. Resumo científico. Disponível em: https://iris.paho.org/handle/ro665.2/52472. Acessado em: 03 de outubro de 2021. 
RAMIREDDY, A.; CHUGH. H.; REINIER, K. et al. (2020). Experience With Hydroxychloroquine and Azithromycin in the Coronavirus Disease 2019 Pandemic: Implications for QT Interval Monitoring. Journal of the American Heart Association. v. 9, n. 12. https://doi.org/ro.116I/JAHA.120.017144

ROSENBERG, E. S.; DUFORT, E. M.; TOMOKO UDO, T. ET AL. (2020). Association of Treatment With Hydroxychloroquine or Azithromycin With InHospital Mortality in Patients With COVID-r9 in New York State. JAMA. v. 323, n. 24, p. 2493-2502. https://doi.org/doi:Io.Iool/jama.2020.8630

SOUZA, A. S. R.; AMORIM, M. M. R.; MELO, A. S. O. et al. (202I). Aspectos gerais da pandemia de COVID-19. Revista Brasileira de Saúde Materno Infantil. V. 2I, n. I, p. 47-64.

VANZELER, M. L. A.; DIAS, F. F.; ANDRADE, F. et al. (202I). Utilização De Azitromicina Para O Tratamento Da Covid-ı: Uma Visão Crítica. Revista Científica Multidisciplinar Núcleo do Conhecimento. v. o9, n. 4, p. 05-2I. https://doi.org/ı0.32749/nucleodoconhecimento.com.br/saude/utilizacao-deazitromicina

VIEIRA, L. M. F, EMERY, E. ANDRIOLO, A. (2020). COVID-i9: laboratory diagnosis for clinicians. An updating article. Sao Paulo Medical Journal. v. 138, n. o3. https://doi.org/10.1590/1516-3180.2020.0240.14052020

WU, T. C.; SACILOTTO, L.; DARRIEUX. F. C. C. et al. (2020). Controle do Intervalo QT para Prevenção de Torsades de Pointes Durante uso de Hidroxicloroquina e/ou Azitromicina em Pacientes com COVID 19. Arquivos Brasileiros de Cardiologia. v. II4, n. 6, p. Io6ı-ı666. https://doi.org/ro.3666o/abc.20200389 